\title{
Effect of Rhodophyta extracts on in vitro ruminal fermentation characteristics, methanogenesis and microbial populations
}

\author{
Shin Ja Lee ${ }^{1, a}$, Nyeon Hak Shin ${ }^{2, a}$, Jin Suk Jeong ${ }^{3}$, Eun Tae Kim ${ }^{4}$, Su Kyoung Lee ${ }^{1}$, and Sung Sill Lee ${ }^{1,3, *}$
}

\footnotetext{
* Corresponding Author: Sung Sill Lee Tel: +82-55-772-1883, Fax: +82-55-772-1889,

E-mail: Iss@gnu.ac.kr

${ }^{1}$ Institute of Agriculture and Life Science \& UniversityCentered Labs, Gyeongsang National University, Jinju 52828, Korea

${ }^{2}$ Livestock Experiment Station, Gyeongsangnamdo Livestock Promotion Research Institute, Sancheong 52733, Korea

${ }^{3}$ Division of Applied Life Science (BK21 program) and Institute of Agriculture \& Life Science (IALS), Gyeongsang National University, Jinju 52828, Korea ${ }^{4}$ National Institute of Animal Science, RDA, Cheonan 31000, Korea

a These authors contributed equally to the work.
}

ORCID

Shin Ja Lee

https://orcid.org/0000-0002-4224-1211

Nyeon Hak Shin

https://orcid.org/0000-0002-9069-0601

Jin Suk Jeong

https://orcid.org/0000-0001-6877-3067

Eun Tae Kim

https://orcid.org/0000-0001-7486-5638

Su Kyoung Lee

https://orcid.org/0000-0002-0997-2899

Sung Sill Lee

https://orcid.org/0000-0002-4621-4333

Submitted Aug 21, 2017; Revised Oct 2, 2017; Accepted Nov 4, 2017
Objective: Due to the threat of global warming, the livestock industry is increasingly interested in exploring how feed additives may reduce anthropogenic greenhouse gas emissions, especially from ruminants. This study investigated the effect of Rhodophyta supplemented bovine diets on in vitro rumen fermentation and rumen microbial diversity.

Methods: Cannulated Holstein cows were used as rumen fluid donors. Rumen fluid:buffer (1:2; $15 \mathrm{~mL}$ ) solution was incubated for up to $72 \mathrm{~h}$ in six treatments: a control (timothy hay only), along with substrates containing 5\% extracts from five Rhodophyta species (Grateloupia lanceolata [Okamura] Kawaguchi, Hypnea japonica Tanaka, Pterocladia capillacea [Gmelin] Bornet, Chondria crassicaulis Harvey, or Gelidium amansii [Lam.] Lamouroux).

Results: Compared with control, Rhodophyta extracts increased cumulative gas production after 24 and $72 \mathrm{~h}(\mathrm{p}=0.0297$ and $\mathrm{p}=0.0047)$. The extracts reduced methane emission at 12 and $24 \mathrm{~h}(\mathrm{p}<0.05)$. In particular, real-time polymerase chain reaction analysis indicated that at $24 \mathrm{~h}$, ciliate-associated methanogens, Ruminococcus albus and Ruminococcus flavefaciens decreased at $24 \mathrm{~h}(\mathrm{p}=0.0002, \mathrm{p}<0.0001$, and $\mathrm{p}<0.0001)$, while Fibrobacter succinogenes (F. succinogenes) increased $(\mathrm{p}=0.0004)$. Additionally, Rhodophyta extracts improved acetate concentration at 12 and $24 \mathrm{~h}(\mathrm{p}=0.0766$ and $\mathrm{p}=0.0132)$, as well as acetate/propionate $(\mathrm{A} / \mathrm{P})$ ratio at 6 and $12 \mathrm{~h}(\mathrm{p}$ $=0.0106$ and $\mathrm{p}=0.0278$ ).

Conclusion: Rhodophyta extracts are a viable additive that can improve ruminant growth performance (higher total gas production, lower A/P ratio) and methane abatement (less ciliateassociated methanogens, Ruminococcus albus and Ruminococcus flavefaciens and more F. succinogenes.

Keywords: Growth Rate; In vitro; Rumen Fermentation; Methane; Rhodophyta

\section{INTRODUCTION}

The past decade has seen a rapid rise in anthropogenic greenhouse gas (GHG) emissions. Notably, methane gas has a global warming potential 25 times greater than carbon dioxide [1], and its concentrations has increased twofold since the early 1800s [2]. Agricultural activities are a major source of methane emission. In particular, ruminant livestock is responsible for $25 \%$ of atmospheric methane globally [3], representing a loss of gross energy intake that could reach $15 \%$ depending on feeding intensity, diet composition, and digestibility [3]. Therefore, animal nutritionists are extremely interested in manipulating the rumen microbial ecosystem to reduce methane emission without adverse effects on rumen function. Specifically, research is needed to identify feed additives that can modify ruminal fermentation characteristics and increase feed use efficiency, thereby inhibiting ruminal methanogenesis. Existing studies focused on determining the potential of feed additives in reducing livestock GHG emissions, while also improving feed use, diet digestibility, and ultimately livestock productivity [4]. Potential feed additives include halogenated analogues, monensin, and a range of plant compounds, such as essential oils, saponins, tannins, and various 
secondary metabolites [5]. Successful dietary changes would be beneficial for individual livestock producers and the industry.

One promising source for feed additives is algae, already economically important due to its current applications in human foods [6] and animal feed [7]. Algae exist in a wide range of forms, broadly classified according to size (micro- versus macroalgae) and primary pigments (green, red, or brown). In particular, macroalgae are a rich source of compounds essential for metabolic function, including various minerals, vitamins, proteins, lipids, and polysaccharides; thus, macroalgae additives can increase basal feed quality [8], animal growth rates, and feed conversion efficiency [9], as well as reduce enteric methane emission [10] in ruminants. Novel food ingredients [11] and many bioactive compounds [12] have been described in reports on algal composition and properties. However, few studies have determined exactly how algae feed additives influence fermentation characteristics and methane mitigation.

Therefore, our objective here was to investigate the effect of using Rhodophyta extracts as a dietary supplement on in vitro ruminal fermentation parameters and methane emission. In vitro techniques were used to allow for rapid screening of fermentation kinetics. Five algal species were compared against a control (noadditive) basal diet, including Grateloupia lanceolata (Okamura) Kawaguchi, Hypnea japonica Tanaka, Pterocladia capillacea (Gmelin) Bornet, Chondria crassicaulis Harvey, and Gelidium amansii (Lam.) Lamouroux.

\section{MATERIALS AND METHODS}

All experimental protocols were approved by the Animal Care and Use Committee of Gyeongsang National University (Jinju, Gyeongsangnam-do, Korea).

\section{Preparation of Rhodophyta extracts}

All algae extracts were obtained from the Jeju Biodiversity Research Institute (JBRI, Jeju, Korea) (Table 1). Each plant was washed, cut into small pieces, freeze-dried, and ground into powder. The powder was than extracted with $70 \%$ or $80 \%$ methyl alcohol, using an ultrasonic cleaner (Branson Ultrasonics corporation, Danbury, CT, USA) at room temperature. After extraction, eluates were filtered through Whatman No. 1 filter paper and concentrated under vacuum. Plant extracts were re-dissolved in dimethyl sulfoxide (Sigma-Aldrich Chemical Co., St. Louis, MO, USA) and diluted using culture media before experimentation.

\section{In vitro fermentation design}

One cannulated Holstein cows $(450 \pm 30 \mathrm{~kg})$ was used as rumen fluid donors and provided with ad libitum access to a mineralvitamin block and water. Twice daily (09:00 and 17:00), cows were fed $2 \%$ of their body weight in timothy hay and commercial concentrate at a 60:40 (w/w) ratio. Rumen fluid was collected before morning feedings and filtered through four layers of cheesecloth. Next, it was diluted with artificial saliva and stored at $39^{\circ} \mathrm{C}$.

The chemical composition (\% dry matter [DM] basis) of commercial timothy hay was as follows: moisture content, $8.87 \%$; crude protein, $13.37 \%$; ether extracts, $2.25 \%$; crude fiber, $21.87 \%$; crude ash, $8.62 \%$; neutral detergent fiber, $53.18 \%$; and acid detergent fiber, $30.57 \%$.

The Rumen fluid was mixed with McDougall's buffer in a 2:1 ratio. Next, $15 \mathrm{~mL}$ of the mixture was dispensed anaerobically into $50-\mathrm{mL}$ serum bottles containing $0.3 \mathrm{~g}$ of timothy substrate and one of five Rhodophyta extracts (5\% of substrate). Bottles were sealed anaerobically with an aluminum-capped butyl rubber stopper in pure $\mathrm{N}_{2}$ gas, and incubated in a shaking incubator (Jeio Tech, SI-900R, Daejeon, Korea; $120 \times \mathrm{rpm}$ ) at $39^{\circ} \mathrm{C}$ for $72 \mathrm{~h}$. The in vitro fermentation experiment was a completely randomized block design and performed in triplicate, using 126 serum bottles ( 6 treatments $\times 7$ incubation times $\times 3$ replicates times).

\section{Analysis of gas profiles and ruminal fermentation characteristics}

Total gas production in the samples was measured with head space gas chromatography using a detachable pressure transducer and a digital readout voltmeter (Laurel Electronics, Inc., Costa Mesa, CA, USA). The transducer was connected to the inlet of a disposable Luer-lock three-way stopcock. Gas pressure in the headspace above the culture medium was read from the LED display unit after inserting a hypodermic syringe needle. Methane and carbon dioxide content was measured using a TCD detector with a Carboxen-1006 Plot capillary column $(30 \mathrm{~mm} \times 0.53 \mathrm{~mm}$, Supelco, Bellefonte, PA, USA), after connecting another stopcock outlet to a gas chromatograph (HP 5890, Agilent Technologies, Santa Clara, CA, USA).

Next, serum bottles were uncapped and the culture medium was subsampled for $\mathrm{pH}$ (MP230, Mettler-Toledo, Columbus, $\mathrm{OH}$, USA), ammonia-N and volatile fatty acid (VFA) analyses. Am-

Table 1. General information on Rhodophyta extracts used in the experiment ${ }^{1)}$

\begin{tabular}{llll}
\hline Stock No. & Scientific name & Family name & Part \\
\hline JBRI-10356 & Grateloupia lanceolata (Okamura) Kawaguchi & Halymeniaceae & Whole plant \\
JBRI-20219 & Hypnea japonica Tanaka & Hypneaceae & Whole plant \\
JBRI-20027 & Pterocladia capillacea (Gmelin) Bornet & Gelidiaceae & Whole plant \\
JBRI-20440 & Chondria crassicaulis Harvey & Rhodomelaceae & Whole plant \\
JBRI-10244 & Gelidium amansii (Lamouroux) Lamouroux & Gelidiaceae & Whole plant \\
\hline
\end{tabular}

\footnotetext{
1) Plant extracts were obtained from Jeju Biodiversity Research institute (JBRI, Jeju, Korea) and data were provided by JBRI.
} 
monia-N concentration was measured as optical density (OD) values at $630 \mathrm{~nm}$ using a UV/VIS spectrophotometer (Model 680, Bio-Rad laboratories, Hercules, CA, USA). For VFA measurements, sub-samples were centrifuged at 3,000 $\times$ rpm for $3 \mathrm{~min}$. The resultant supernatant was filtered using a $0.2 \mu \mathrm{m}$ disposable syringe filter (Whatman Inc., Clifton, NJ, USA) high performance liquid chromatography (Agilent-1200, Waldbronn, Germany) using a UV/VIS detector with a MetaCarb 87H column (300 mm× $7.8 \mathrm{~mm}$, Varian, Palo Alto, CA, USA).

In vitro DM disappearance rate was determined following a modified Ørskov's method, using nylon-bag digestion. After incubation, the nylon bag containing serum bottles was washed twice in a water-bath equipped with a Heidolph Rotamax 120 (Heidolph Instruments, Nuremberg, Germany) at $100 \times$ rpm for $30 \mathrm{~min}$ and then oven dried at $60^{\circ} \mathrm{C}$ to a constant weight. Dry matter disappearance was the difference in serum-bottle weight before and after incubation.

\section{Microbial growth rate}

At the end of each fermentation period, samples were centrifuged at $3,000 \times \mathrm{rpm}$ for $3 \mathrm{~min}$ to remove feed particles. The supernatant was then re-centrifuged at $14,000 \times \mathrm{rpm}$ for $3 \mathrm{~min}$ to obtain a final supernatants for protein and glucose analysis. Some of the supernatant was dyed with Coomassie Blue G-250 for spectrophotometrically measuring protein content as $\mathrm{OD}$ at 595 nm (Model 680, Bio-Rad Laboratories, USA). For measuring glucose, $200 \mu \mathrm{L}$ of supernatant was mixed with $600 \mu \mathrm{L}$ of DNS solution and incubated for $5 \mathrm{~min}$ in a boiling water bath. Glucose concentration was the OD at $595 \mathrm{~nm}$, determined with a microplate reader (Model 680, Bio-Rad Laboratories, USA). Pellets from the centrifugation were washed with sodium phosphate buffer ( $\mathrm{pH}$ 6.5) four more times and then subjected to OD measurements at $550 \mathrm{~nm}$ (Model 680, Bio-Rad Laboratories, USA) to evaluate microorganism growth rates.

\section{Quantitative real-time polymerase chain reaction}

Samples were placed in screw-capped tubes containing silica beads for DNA extraction with a high-speed reciprocal shaker, following a modified bead-beating protocol with a Soil kit (Macherey-nagel, Düren, Germany). Briefly, a 1.0-mL aliquot of the incubated culture solution was centrifuged at 3,000×rpm, and then placed in a NanoDrop Spectrophotometer (Thermo Scientific, Wilmington, DE, USA) to determine nucleic acid concentrations.

Previous reports provided primers and thermocycling protocols used for amplification of general bacteria [13], ciliate protozoa [14], methanogenic archaea [15], Fibrobacter succinogenes (F. succinogenes) [13], Ruminococcus albus (R. albus) [16] and Ruminococcus flavefaciens ( $R$. flavefaciens) [13].

Quantitative real-time polymerase chain reaction (qPCR) assays (CFX96 Real-Time system; Bio Rad, USA) using the SYBR Green Supermix (QPK-201, Toyobo Co., LTD., Tokyo, Japan) were performed following previously described methods (Denman and
McSweeney [13] and Denman et al [15]). Microbial abundance was expressed with the following equation: relative quantification $=2^{-\Delta \mathrm{Ct}(\text { Target })-\Delta \mathrm{Ct}(\text { Control) }}$, where Ct represents threshold cycle. The qPCR reaction mixtures $(20 \mu \mathrm{L})$ contained forward/ reverse primers, SYBR Green Supermix and DNA template.

\section{Statistical analysis}

All data were analyzed using the general linear model procedure of SAS [17]. Between-treatment differences were examined using Duncan's multiple comparison tests. Orthogonal contrasts were used to test the overall effect of Rhodophyta supplementation (control [CON] vs treatment) and the effect $H$. japonica (control [CON] vs GLK). Data are presented as means \pm standard error of the mean. Significance was set at $\mathrm{p}<0.05$, whereas $\mathrm{p}<0.10$ was considered a tendency.

\section{RESULTS}

\section{In vitro fermentation characteristics}

Compared with CON, Rhodophyta extracts raised $\mathrm{pH}$ at $6,9,12$, and $72 \mathrm{~h}(\mathrm{p}=0.0006, \mathrm{p}=0.0330, \mathrm{p}=0.0004$, and $\mathrm{p}=0.0136)$, while GLK raised $\mathrm{pH}$ at 6 , and $12 \mathrm{~h}(\mathrm{p}=0.0006$ and $\mathrm{p}=0.0004)$ (Table 2). Rhodophyta extracts and GLK decreased cumulative gas production by ruminal microbes at $3 \mathrm{~h}(\mathrm{p}=0.0001$ and $\mathrm{p}=$ $0.0016)$ and $6 \mathrm{~h}(\mathrm{p}=0.0149$ and $\mathrm{p}=0.0536)$, but increased production at $24 \mathrm{~h}$ while HJT (and p $=0.0058)$ and $72 \mathrm{~h}(\mathrm{p}=0.0047$ and $\mathrm{p}=0.0133)$. Except at $72 \mathrm{~h}(\mathrm{p}=0.0249)$, none of the Rhodophyta extracts significantly affected DM disappearance during the experiment (Table 2).

Rhodophyta extracts and GLK reduced methane emissions relative to CON at $12 \mathrm{~h}(\mathrm{p}=0.0077$ and $\mathrm{p}=0.0306)$ and $24 \mathrm{~h}(\mathrm{p}$ $=0.0008$ and $\mathrm{p}=0.0183$ ) (Table 3 ). Furthermore, at $9 \mathrm{~h}$ Rhodophyta extracts were not reduced carbon dioxide, but ammonia reduce $(\mathrm{p}=0.0440$ and $\mathrm{p}=0.0419)$, while GLK reduced ammonia $(\mathrm{p}=0.0414)$ (Table 3$)$.

Rhodophyta extracts improved acetate concentration over $\mathrm{CON}$ at 12 and $24 \mathrm{~h}(\mathrm{p}=0.0766$ and $\mathrm{p}=0.0132)$, while GLK exerted the same effect at $24 \mathrm{~h}(\mathrm{p}=0.0187)$ (Table 4$)$. Except at $12 \mathrm{~h}$ ( $p=0.0659$ ), Rhodophyta extracts did not significantly differ from $\mathrm{CON}$ in effects on propionate concentration. Overall, Rhodophyta extracts and GLK improved A/P ratio at $6 \mathrm{~h}(\mathrm{p}=0.0106$ and $\mathrm{p}$ $=0.0118)$ and $12 \mathrm{~h}(\mathrm{p}=0.0278$ and $\mathrm{p}=0.0398)$.

\section{In vitro ruminal change in microbial diversity}

Rhodophyta extracts reduced microbial growth rate compared with CON at $6 \mathrm{~h}(\mathrm{p}=0.0020$ and $\mathrm{p}=0.0072)$, but GLK increased growth rate at $24 \mathrm{~h}(\mathrm{p}=0.0247)$ (Table 5). Additionally, both Rhodophyta extracts and GLK increased protein concentration at 9 h expect to HJT, PCB extracts $(p=0.0298$ and $p=0.0711)$. PCB extract, glucose concentration significantly decreased at $3 \mathrm{~h}(\mathrm{p}$ $=0.0673$ ), then increased at $24 \mathrm{HJT}, \mathrm{PCB}$ extracts and $48 \mathrm{~h}$ PCB extract ( $\mathrm{p}=0.0163$ and $\mathrm{p}=0.0867)$; glucose also increased under 
Table 2. Effects of Rhodophyta extracts on in vitro rumen microbial fermentation, specifically cumulative pH, gas production, and dry matter (DM) disappearance

\begin{tabular}{|c|c|c|c|c|c|c|c|c|c|}
\hline \multirow{2}{*}{ Incubation times (h) } & \multirow{2}{*}{ CON } & \multicolumn{5}{|c|}{ Treatments $^{1)}$} & \multirow{2}{*}{ SEM } & \multicolumn{2}{|c|}{ Contrast } \\
\hline & & GLK & HJT & PCB & $\mathrm{CCH}$ & GAL & & CON vs Others & CON vs HJT \\
\hline \multicolumn{10}{|l|}{ pH } \\
\hline 3 & $7.35^{c}$ & $7.35^{c}$ & $7.42^{b}$ & $7.44^{a b}$ & $7.46^{\mathrm{a}}$ & $7.47^{\mathrm{a}}$ & 0.02 & 0.0003 & 0.0057 \\
\hline 6 & $7.29^{c}$ & $7.37^{\mathrm{b}}$ & $7.38^{\mathrm{b}}$ & $7.42^{\mathrm{ab}}$ & $7.46^{\mathrm{a}}$ & $7.44^{\mathrm{ab}}$ & 0.04 & 0.0006 & 0.0255 \\
\hline 9 & $7.12^{c}$ & $7.24^{\mathrm{abc}}$ & $7.17^{\mathrm{bc}}$ & $7.22^{\mathrm{abc}}$ & $7.29^{\mathrm{ab}}$ & $7.34^{\mathrm{a}}$ & 0.08 & 0.0330 & 0.5367 \\
\hline 12 & $7.00^{c}$ & $7.01^{\mathrm{c}}$ & $7.14^{b}$ & $7.23^{\mathrm{ab}}$ & $7.28^{\mathrm{a}}$ & $7.29^{\mathrm{a}}$ & 0.05 & 0.0004 & 0.0139 \\
\hline 24 & $6.46^{\mathrm{ab}}$ & $6.39^{b}$ & $6.37^{b}$ & $6.52^{\mathrm{ab}}$ & $6.51^{\mathrm{ab}}$ & $6.60^{\mathrm{a}}$ & 0.10 & 0.7227 & 0.3162 \\
\hline 48 & $6.20^{\mathrm{ab}}$ & $6.15^{b}$ & $6.16^{a b}$ & $6.23^{\mathrm{ab}}$ & $6.26^{a}$ & $6.25^{a b}$ & 0.05 & 0.7995 & 0.4314 \\
\hline 72 & $6.12^{b}$ & $6.15^{a b}$ & $6.16^{\mathrm{ab}}$ & $6.20^{\mathrm{a}}$ & $6.19^{\mathrm{a}}$ & $6.19^{\mathrm{a}}$ & 0.05 & 0.0136 & 0.1591 \\
\hline \multicolumn{10}{|c|}{ Total gas production (mL/g DM) } \\
\hline 3 & $151.20^{\mathrm{a}}$ & $148.29^{b}$ & $146.97^{b c}$ & $145.50^{c}$ & $145.66^{c}$ & $143.28^{d}$ & 1.15 & 0.0001 & 0.0016 \\
\hline 6 & $161.97^{\mathrm{a}}$ & $152.84^{b}$ & $152.31^{b}$ & $151.15^{b}$ & $150.88^{b}$ & $150.14^{b}$ & 2.75 & 0.01492 & 0.0536 \\
\hline 9 & $167.72^{a b}$ & $165.03^{b c}$ & $165.61^{\mathrm{abc}}$ & $169.57^{\mathrm{a}}$ & $164.61^{b c}$ & $162.87^{c}$ & 2.16 & 0.0962 & 0.1194 \\
\hline 12 & 177.86 & 179.29 & 176.54 & 177.39 & 175.70 & 174.28 & 3.42 & 0.5062 & 0.5777 \\
\hline 24 & $238.43^{b}$ & $249.20^{\mathrm{ab}}$ & $252.84^{\mathrm{a}}$ & $248.19^{a b}$ & $241.91^{\mathrm{ab}}$ & $240.54^{\mathrm{ab}}$ & 6.48 & 0.0297 & 0.0058 \\
\hline 48 & 260.92 & 268.53 & 267.47 & 260.76 & 261.08 & 263.35 & 5.16 & 0.4663 & 0.2106 \\
\hline 72 & $271.75^{b}$ & $279.61^{\mathrm{a}}$ & $279.09^{a}$ & $281.93^{\mathrm{a}}$ & $276.5^{\mathrm{ab}}$ & $275.65^{\mathrm{ab}}$ & 3.59 & 0.0047 & 0.0133 \\
\hline \multicolumn{10}{|l|}{ DM disappearance (\%) } \\
\hline 3 & 21.03 & 20.54 & 21.62 & 24.39 & 21.46 & 21.39 & 2.33 & 0.5596 & 0.7541 \\
\hline 6 & 22.25 & 22.32 & 22.42 & 22.35 & 22.83 & 22.15 & 0.85 & 0.7418 & 0.7926 \\
\hline 9 & 24.31 & 24.80 & 24.40 & 26.53 & 24.95 & 25.71 & 1.45 & 0.3473 & 0.9440 \\
\hline 12 & 28.08 & 27.89 & 27.39 & 26.67 & 26.68 & 27.07 & 1.34 & 0.2044 & 0.4591 \\
\hline 24 & 38.40 & 41.02 & 40.41 & 40.67 & 41.98 & 38.56 & 2.65 & 0.2231 & 0.3639 \\
\hline 48 & 47.57 & 48.25 & 48.24 & 46.36 & 47.35 & 44.97 & 1.88 & 0.6467 & 0.6529 \\
\hline 72 & $51.08^{\mathrm{a}}$ & $48.69^{\mathrm{ab}}$ & $49.33^{\mathrm{ab}}$ & $49.65^{a b}$ & $46.97^{b}$ & $48.13^{a b}$ & 1.74 & 0.0249 & 0.1872 \\
\hline
\end{tabular}

SEM, standard error of the mean; DM, dry matter.

1) Dietary treatments were as follows: CON, basal diet (without Rhodophyta extracts); GLK, 5\% Grateloupia lanceolata (Okamura) Kawaguchi; HJT, 5\% Hypnea japonica Tanaka; PCB, 5\% Pterocladia capillacea

(Gmelin) Bornet; CCH, Chondria crassicaulis Harvey; GAL, 5\% Gelidium amansii (Lam.) Lamouroux percentages are based on substrate (timothy hay) amount.

abc Means with different superscripts in the same row indicate significant differences $(p<0.05)$.

Table 3. Effect of Rhodophyta extracts on methane, carbon dioxide, and ammonia emissions during in vitro mixed rumen microbial fermentation

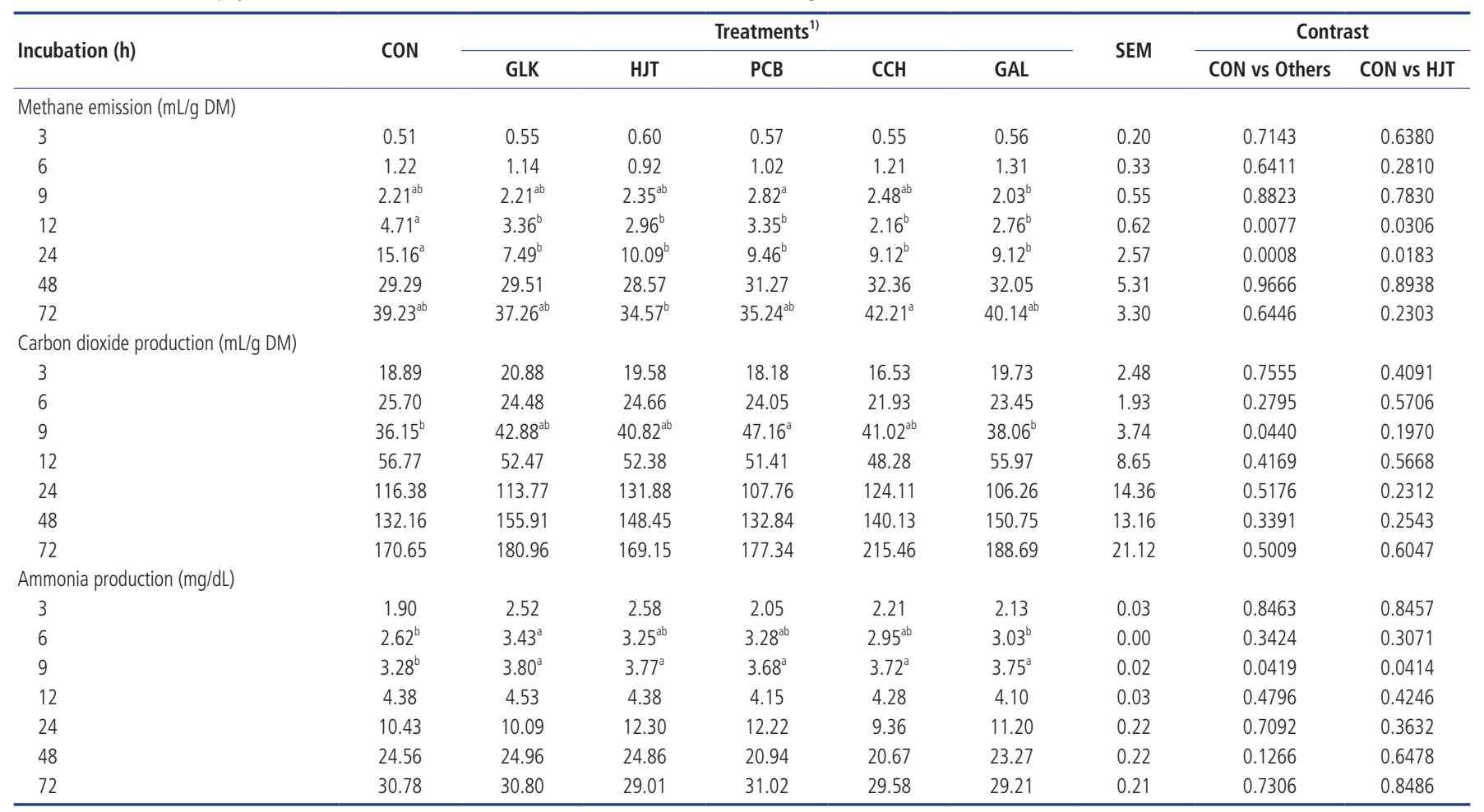

SEM, standard error of the mean; DM, dry matter.

1) Dietary treatments were as follows (percent basis of timothy substrate): CON, basal diet (timothy without Rhodophyta extracts); GLK, 5\% Grateloupia lanceolate (Okamura) Kawaguchi; HJT, $5 \%$ Hypnea japonica Tanake; PCB, 5\% Pterocladia capillacea (Gmelin) Bornet; CCH, Chondria crassicaulis Harvey; GAL, Gelidium amansii (Lam.) Lamouroux.

abc Means with different superscripts in the same row indicate significant differences significantly $(p<0.05)$. 
Table 4. Effects of Rhodophyta extracts on acetic acid, propionic acid, and acetic acid/propionic acid (A/P) ratio during mixed rumen microbial fermentation

\begin{tabular}{|c|c|c|c|c|c|c|c|c|c|}
\hline \multirow{2}{*}{ Incubation (h) } & \multirow{2}{*}{$\mathrm{CON}$} & \multicolumn{5}{|c|}{ Treatments ${ }^{1)}$} & \multirow{2}{*}{ SEM } & \multicolumn{2}{|c|}{ Contrast } \\
\hline & & GLK & HJT & PCB & $\mathrm{CCH}$ & GAL & & CON vs Others & CON vs HJT \\
\hline \multicolumn{10}{|c|}{ Acetic acid concentration (mM/g) } \\
\hline 3 & 25.41 & 25.70 & 25.55 & 25.48 & 25.95 & 24.60 & 1.75 & 0.9356 & 0.6172 \\
\hline 6 & $27.37^{b c}$ & $28.52^{a b}$ & $28.52^{\mathrm{ab}}$ & $29.76^{\mathrm{a}}$ & $29.05^{\mathrm{ab}}$ & $26.50^{c}$ & 0.89 & 0.4376 & 0.7281 \\
\hline 9 & $28.76^{c}$ & $33.40^{\mathrm{a}}$ & $30.43^{b c}$ & $32.27^{\mathrm{ab}}$ & $33.31^{\mathrm{a}}$ & $31.90^{\mathrm{ab}}$ & 1.38 & 0.2816 & 0.3021 \\
\hline 12 & $31.09^{c}$ & $42.37^{\mathrm{a}}$ & $33.94^{\mathrm{bc}}$ & $34.49^{b c}$ & $35.76^{b}$ & $36.63^{b}$ & 2.25 & 0.0766 & 0.4842 \\
\hline 24 & $52.98^{b}$ & $57.00^{\mathrm{a}}$ & $59.34^{\mathrm{a}}$ & $57.81^{\mathrm{a}}$ & $57.35^{\mathrm{a}}$ & $56.96^{\mathrm{a}}$ & 2.25 & 0.0132 & 0.0187 \\
\hline 48 & $67.14^{\mathrm{abc}}$ & $68.43^{\mathrm{ab}}$ & $70.92^{\mathrm{a}}$ & $62.90^{c}$ & $64.52^{b c}$ & $62.89^{c}$ & 2.82 & 0.4979 & 0.1629 \\
\hline 72 & 75.88 & 80.48 & 76.35 & 73.04 & 79.66 & 75.06 & 3.80 & 0.7305 & 0.9382 \\
\hline \multicolumn{10}{|c|}{ Propionic acid concentration $(\mathrm{mM} / \mathrm{g})$} \\
\hline 3 & 4.51 & 4.85 & 4.71 & 6.06 & 5.29 & 5.78 & 1.75 & 0.2197 & 0.4275 \\
\hline 6 & $6.77^{\mathrm{b}}$ & $8.07^{a b}$ & $9.99^{\mathrm{a}}$ & $9.86^{\mathrm{a}}$ & $9.79^{\mathrm{a}}$ & $9.44^{\mathrm{a}}$ & 1.00 & 0.7694 & 0.4424 \\
\hline 9 & 8.95 & 12.34 & 12.33 & 10.99 & 11.95 & 12.14 & 2.76 & 0.1731 & 0.1583 \\
\hline 12 & $11.20^{c}$ & $24.20^{\mathrm{a}}$ & $19.90^{\mathrm{ab}}$ & $17.86^{\mathrm{ab}}$ & $18.93^{\mathrm{ab}}$ & $15.57^{\text {bc }}$ & 3.37 & 0.0659 & 0.3819 \\
\hline 24 & $25.11^{b c}$ & $28.87^{\mathrm{a}}$ & $26.76^{\mathrm{b}}$ & $23.86^{c}$ & $26.34^{b}$ & $26.28^{b}$ & 0.87 & 0.3991 & 0.4483 \\
\hline 48 & $28.11^{\mathrm{ab}}$ & $29.87^{\mathrm{a}}$ & $27.85^{\mathrm{ab}}$ & $26.41^{b}$ & $29.26^{a}$ & $28.09^{a b}$ & 1.34 & 0.9863 & 0.1338 \\
\hline 72 & $29.41^{b c}$ & $34.96^{\mathrm{a}}$ & $32.03^{a b}$ & $27.21^{c}$ & $33.49^{\mathrm{a}}$ & $31.26^{\mathrm{ab}}$ & 2.07 & 0.7666 & 0.9417 \\
\hline \multicolumn{10}{|l|}{ A/P ratio } \\
\hline 3 & 5.63 & 5.63 & 5.42 & 4.70 & 5.54 & 4.70 & 1.53 & 0.1198 & 0.5389 \\
\hline 6 & $4.04^{\mathrm{a}}$ & $3.53^{\mathrm{a}}$ & $2.88^{b}$ & $3.07^{b}$ & $2.98^{b}$ & $2.84^{\mathrm{b}}$ & 0.39 & 0.0106 & 0.0118 \\
\hline 9 & 3.23 & 3.23 & 2.50 & 2.94 & 2.81 & 2.66 & 0.58 & 0.4799 & 0.7381 \\
\hline 12 & $2.78^{\mathrm{a}}$ & $1.82^{b}$ & $1.81^{\mathrm{b}}$ & $1.96^{\mathrm{b}}$ & $1.91^{\mathrm{b}}$ & $2.37^{\mathrm{ab}}$ & 0.33 & 0.0278 & 0.0398 \\
\hline 24 & $2.11^{b c}$ & $1.97^{\mathrm{c}}$ & $2.22^{\mathrm{b}}$ & $2.43^{\mathrm{a}}$ & $2.18^{b c}$ & $2.17^{\mathrm{bc}}$ & 0.11 & 0.0301 & 0.0996 \\
\hline 48 & $2.39^{\mathrm{ab}}$ & $2.39^{b}$ & $2.55^{\mathrm{a}}$ & $2.38^{\mathrm{ab}}$ & $2.21^{b}$ & $2.24^{b}$ & 0.11 & 0.4668 & 0.5251 \\
\hline 72 & 2.58 & 2.58 & 2.40 & 2.69 & 2.38 & 2.41 & 0.20 & 0.9440 & 0.9072 \\
\hline
\end{tabular}

SEM, standard error of the mean; A/P, acetate/propionate.

1) Dietary treatments were as follows (percent basis of timothy substrate): CON, basal diet (timothy without Rhodophyta extracts); GLK, 5\% Grateloupia lanceolata (Okamura) Kawaguchi; HJT, 5\% Hypnea japonica Tanaka; PCB, 5\% Pterocladia capillacea (Gmelin) Bornet; CCH, 5\% Chondria crassicaulis Harvey; GAL, 5\% Gelidium amansii (Lam.) Lamouroux.

${ }^{a b c}$ Means with different superscripts in the same row differ significantly $(p<0.05)$.

GLK at $24 \mathrm{~h}(\mathrm{p}=0.0070)($ Table 5$)$.

Compared with CON, Rhodophyta supplementation decreased the ciliate-associated methanogen population at $24 \mathrm{~h}(\mathrm{p}$ $=0.0002$; Figure 1), but not the Methanogenic archaea population Rhodophyta extracts also significantly reduced $R$. albus at 12 and $24 \mathrm{~h}(\mathrm{p}=0.0860$ and $\mathrm{p}<0.0001)$, as well as $R$. flavefaciens at $24 \mathrm{~h}$ $(\mathrm{p}<0.0001)$ (Figure 1). Furthermore, F. succinogenes populations increased at 12 and $24 \mathrm{~h}(\mathrm{p}=0.0049$ and $\mathrm{p}=0.0004)$ after Rhodophyta treatments (Figure 1).

\section{DISCUSSION}

This study investigated the effects of five Rhodophyta species on in vitro fermentation characteristics and changes to ruminal of microbial diversity. Below, we discussed the implications of our results in the context of previous research.

\section{In vitro fermentation characteristics}

Overall, $\mathrm{pH}$ remained consistently within 6.12 and 7.47 across Rhodophyta-extract treatments. Interestingly, $\mathrm{CON}$ had the lowest post-fermentation $\mathrm{pH}$, suggesting that Rhodophyta supplementation creates a more alkaline environment during microbial fermentation. Because a $\mathrm{pH}$ range of 5.0 to 7.8 is ideal for ruminal microbial activity [18], the algal extracts likely had a negative effect.

Dietary fiber from Rhodophyta increased total gas production without any difference in DM disappearance rate. Overall, DM disappearance did not significantly differ across time points and Rhodophyta species, except with Chondria crassicaulis at 72 h. Additionally, total gas production of all Rhodophyta-extract treatments was significantly higher than CON only at 24 and 72 $\mathrm{h}$ incubation, hinting at a strategy for improving feed efficiency. Similarly interesting effects of dietary fiber in algae have been previously reported [19]. Overall, all tested Rhodophyta extracts have the potential to improve gas production and fermentation management, thus assisting in ruminant feeding.

Although the use of terrestrial plants to manipulate enteric methane emission have been extensively investigated [3], our study is the first to provide evidence that Rhodophyta extracts can effectively reduce in vitro methane emission and alter rumen microbial diversity. Thus, our data supports the hypothesis put forth by several previous reserarchers [20]. All Rhodophyta extracts significantly reduced methane emission after 12 and $24 \mathrm{~h}$ of incubation, with $H$. japonica having the strongest effect. Re- 
Table 5. Effects of Rhodophyta extracts on rumen microbial growth rate, as well as protein and glucose concentrations

\begin{tabular}{|c|c|c|c|c|c|c|c|c|c|}
\hline \multirow{2}{*}{ Incubation (h) } & \multirow{2}{*}{ CON } & \multicolumn{5}{|c|}{ Treatments ${ }^{1)}$} & \multirow{2}{*}{ SEM } & \multicolumn{2}{|c|}{ Contrast } \\
\hline & & GLK & HJT & PCB & $\mathrm{CCH}$ & GAL & & CON vs Others & CON vs HJT \\
\hline \multicolumn{10}{|c|}{ Microbial growth rate (OD at $550 \mathrm{~nm})$} \\
\hline 6 & $0.20^{\mathrm{a}}$ & $0.20^{\mathrm{a}}$ & $0.16^{\mathrm{b}}$ & $0.16^{b}$ & $0.14^{b}$ & $0.16^{b}$ & 0.02 & 0.0020 & 0.0072 \\
\hline 9 & 0.28 & 0.32 & 0.31 & 0.28 & 0.31 & 0.32 & 0.03 & 0.1542 & 0.2201 \\
\hline 12 & 0.39 & 0.40 & 0.40 & 0.43 & 0.37 & 0.42 & 0.10 & 0.4690 & 0.6618 \\
\hline 72 & 0.47 & 0.46 & 0.41 & 0.49 & 0.42 & 0.45 & 0.05 & 0.5029 & 0.2191 \\
\hline \multicolumn{10}{|c|}{ Protein concentration (mM/g) } \\
\hline 3 & 0.20 & 0.20 & 0.19 & 0.20 & 0.21 & 0.20 & 0.01 & 0.5146 & 0.2318 \\
\hline 6 & 0.20 & 0.19 & 0.20 & 0.19 & 0.19 & 0.20 & 0.01 & 0.2117 & 0.7021 \\
\hline 9 & $0.16^{\mathrm{b}}$ & $0.23^{\mathrm{a}}$ & $0.22^{\mathrm{ab}}$ & $0.16^{b}$ & $0.26^{\mathrm{a}}$ & $0.24^{\mathrm{a}}$ & 0.04 & 0.0298 & 0.0711 \\
\hline \multicolumn{10}{|c|}{ Glucose concentration (mL/mg) } \\
\hline 3 & $0.55^{\mathrm{a}}$ & $0.49^{\mathrm{ab}}$ & $0.48^{\mathrm{ab}}$ & $0.43^{b}$ & $0.51^{\mathrm{ab}}$ & $0.47^{\mathrm{ab}}$ & 0.06 & 0.0673 & 0.1473 \\
\hline 6 & 0.21 & 0.22 & 0.18 & 0.25 & 0.22 & 0.28 & 0.07 & 0.6734 & 0.6699 \\
\hline 9 & 0.17 & 0.17 & 0.18 & 0.17 & 0.16 & 0.16 & 0.02 & 0.8137 & 0.3129 \\
\hline 12 & 0.14 & 0.13 & 0.14 & 0.15 & 0.14 & 0.14 & 0.01 & 0.6933 & 0.6498 \\
\hline 24 & $0.10^{b}$ & $0.12^{\mathrm{a}}$ & $0.12^{\mathrm{a}}$ & $0.12^{\mathrm{a}}$ & $0.10^{b}$ & $0.10^{b}$ & 0.01 & 0.0163 & 0.0070 \\
\hline 48 & $0.09^{b}$ & $0.11^{\mathrm{ab}}$ & $0.10^{\mathrm{ab}}$ & $0.11^{\mathrm{a}}$ & $0.09^{\mathrm{ab}}$ & $0.10^{\mathrm{ab}}$ & 0.01 & 0.0867 & 0.1780 \\
\hline 72 & 0.10 & 0.10 & 0.11 & 0.10 & 0.11 & 0.11 & 0.02 & 0.5719 & 0.4970 \\
\hline
\end{tabular}

SEM, standard error of the mean; OD, optical density.

1) Dietary treatments were as follows (percent basis of timothy substrate): CON, basal diet (timothy without Rhodophyta extracts); GLK, 5\% Grateloupia lanceolata (Okamura) Kawaguchi; HJT, 5\% Hypnea japonica Tanaka; PCB, 5\% Pterocladia capillacea (Gmelin) Bornet; CCH, 5\% Chondria crassicaulis Harvey; GAL, 5\% Gelidium amansii (Lam.) Lamouroux.

${ }^{a b c}$ Means with different superscripts in the same row indicate significant differences $(p<0.05)$.

duced methane emission may have been partially due to alterations in microbial diversity; protozoans (ciliate-associated methanogens) [21] and fibrolytic microbes (R. albus [22]; R. flavefaciens [23]) both decreased, while F. succinogenes increased [21]. R. albus is a very promising candidate for producing $\mathrm{H}_{2}$ from plant forage, because the bacteria can digest cellulosic and hemicellulosic biomass [22]. Likewise, $R$. favefaciens normally produces succinic acid as a major fermentation product together with acetic and formic acids, $\mathrm{H}_{2}$, and carbon dioxide. In contrast, $F$ succinogenes is a non- $\mathrm{H}_{2}$-producing species. A previous study [24] showed that when the dominant fibrolytic species was non- $\mathrm{H}_{2}$-producing, methane emission decreased significantly without impairing fiber degradation and fermentations in the rumen. Together, these results suggest that $\mathrm{H}_{2}$ is critical to the intestinal microbial ecosystem of ruminants. Thus, because hydrogen produced during ruminant enteric fermentation is the precursor of methane emission, regulating of $\mathrm{H}_{2}$ is more critical to controlling ruminant methane emission than regulating methane directly.

Ruminal methanogens primarily use hydrogen and carbon dioxide during methanogenesis, along with formate derived from acetate production. When hydrogen is removed [25], methanogen consumption of carbon dioxide and formate allows fermenta- tion-related microbes to function optimally and support complete substrate oxidation [26]. Our study suggests that Rhodophyta extracts increase carbon dioxide emission at $9 \mathrm{~h}$, as well as acetate concentration at 12 and $24 \mathrm{~h}$. Unfortunately, we could not detect enough hydrogen to in this study to directly test our hypothesis regarding the removal of $\mathrm{H}_{2}$ gas.

Methane emission reduction at 12 and $24 \mathrm{~h}$ can be attributed to increased propionate concentration at $12 \mathrm{~h}$. The increase results in more hydrogen use instead of acetate, thus lowering $\mathrm{A} / \mathrm{P}$ ratios (relative to $\mathrm{CON}$ ) and indicating a clear effect on fermentation. However, the A/P ratios were within optimal fermentation conditions [25]. Rhodophyta extracts resulted in a significant increase in acetate and propionate concentrations and a lower $\mathrm{A} / \mathrm{P}$ ratio than $\mathrm{CON}$ at $12 \mathrm{~h}$ incubation, demonstrating that fermentation was significantly affected. These effects are likely due to secondary metabolites in plants that exert anti-microbial properties. In Rhodophyta specifically, secondary metabolites include terpenes [27] and halogenated compounds [28] that inhibit a wide range of microorganisms. We conclude that the extract dosage used in our study was sufficient to reduce methane emission without seriously affecting nutritionally important fermentation parameters. 
(a)

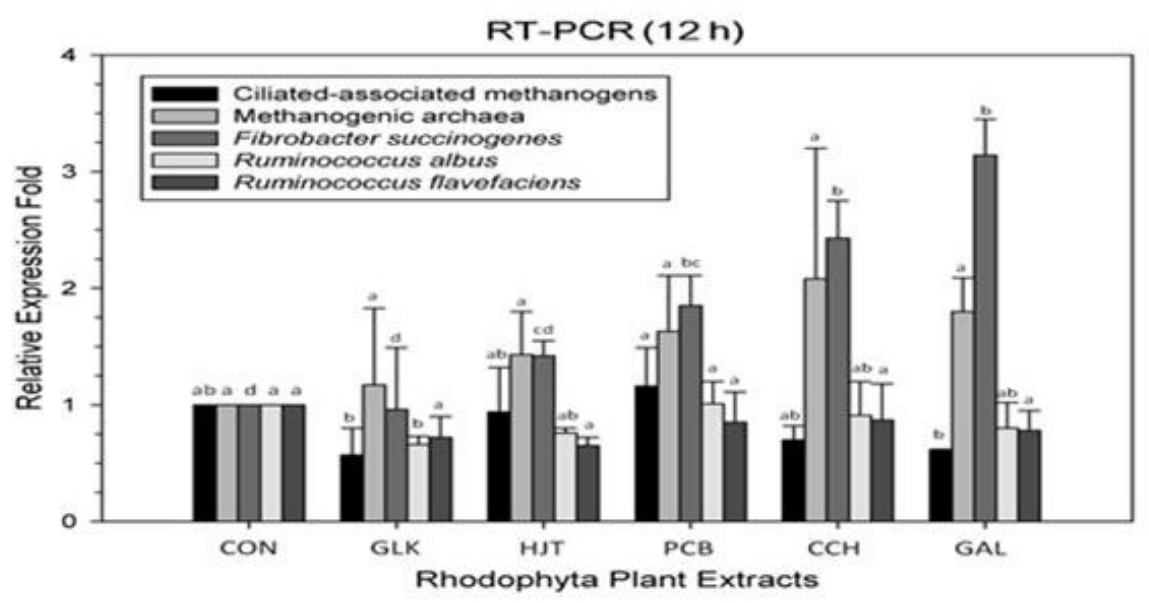

(b)

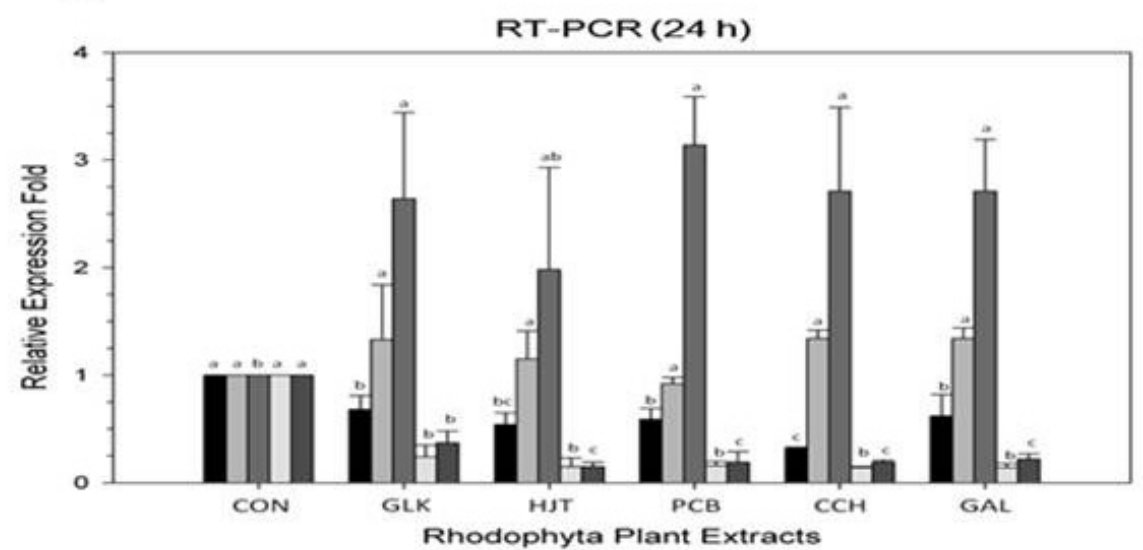

Figure 1. Relative quantification of rumen microbial populations under in vitro ruminal fermentation after $12 \mathrm{~h}(\mathrm{a})$ and $24 \mathrm{~h}$ (b) incubation with various Rhodophyta extracts. Control, no addition; GLK, Grateloupia lanceolata (Okamura) Kawaguchi; HJT, Hypnea japonica Tanaka; PCB, Pterocladia capillacea (Gmelin) Bornet; CCH, Chondria crassicaulis Harvey; GAL, Gelidium amansii (Lam.) Lamouroux. All extracts were $5 \%$ of the substrate(timothy hay) amount. ${ }^{\text {abcd }}$ Means with different superscripts in the same row differ significantly $(p<0.05)$.

\section{Microbial growth rate}

Rhodophyta extracts significantly reduced microbial growth rate at $6 \mathrm{~h}$ but increased at $24 \mathrm{~h}$. This pattern probably occurred because rumen microorganisms were acclimating to changing environmental conditions during the first $6 \mathrm{~h}$, and increased exponentially when they adapted (at around $24 \mathrm{~h}$ ). After $48 \mathrm{~h}$, however, nutrient depletion and increasing amounts of waste likely inhibited microbial growth [18]. Furthermore, we observed that Rhodophyta extract treatments with elevated microbial growth rates also had higher total gas production and lower $\mathrm{pH}$ than $\mathrm{CON}$. This outcome agrees with previous research showing that rumen-microbe growth rate is closely correlated with total gas production and fermentation.

Although rumen ammonia concentration can vary based on feed protein proportions and degradation rate, our use of timothy hay as the sole substrate led to a lack of significant differences in ammonia concentration (except at $6 \mathrm{~h}$ ). Optimal ammonia con- centration for ruminal microbe growth is $8 \mathrm{mg} / \mathrm{dL}$, whereas a concentration $\geq 140 \mathrm{mg} / \mathrm{dL}$ is inhibitory [18]. Maintaining optimal ammonia concentration can improve protein synthesis in most ruminal microorganisms but the two variables are not correlated [29]. Overall, the observed range of ammonia concentrations (1.90 to $30.80 \mathrm{mg} / \mathrm{dL}$ ) is a strong indicator of proper rumen fermentation, with no negative side effects resulting from the Rhodophyta extracts.

Finally, in ruminants, rumen microbial fermentation releases VFAs as the major end products, instead of glucose. Here, we observed the propionate increased significantly at $12 \mathrm{~h}$ and 24 $\mathrm{h}$, correlating with a later glucose increase at $24 \mathrm{~h}$ and $48 \mathrm{~h}$. This outcome in line with the fact that propionate is the most abundant of the glucogenic acids ( $15 \%$ to $40 \%$ of total ruminally released organic acids) and the predominant substrate for gluconeogenesis in ruminants [30], a characteristic that qualitatively distinguishes ruminant and non-ruminant gluconeogenesis 


\section{CONCLUSION}

The results of our study indicate that Rhodophyta extracts are a viable feed additive that can improve ruminant growth performance (increased total gas production and decreased acetate/ propionate ratio) and reduce methane emissions (decreased ciliateassociated methanogens, $R$. albus and R. flavefaciens). Although more research is necessary to clarify the exact effects of specific Rhodophyta on feed intake, feed use efficiency, and methane abatement, we found that Hypnea japonica significantly reduced methane production and had a moderate effect on total gas. These results are promising and suggest that Rhodophyta extracts could mitigate undesirable outcomes of rumen fermentation.

\section{CONFLICT OF INTEREST}

We certify that there is no conflict of interest with any financial organization regarding the material discussed in the manuscript.

\section{ACKNOWLEDGMENTS}

This work was supported by the National Foundation of Korea Grant funded by the Korean Government (NRF-2015R1A6A1A 03031413). Jin Suk Jeong was supported by Postdoctoral Fellowship from the BK 21+ Program, the Ministry of Education, Science and Technology, Republic of Korea. This work was presented as a part of a doctoral dissertation by Nyeon Hak Shin.

\section{REFERENCES}

1. Denman K, Brasseur G, Chidthaisong A, et al. Couplings between changes in the climate system and biogeochemistry. In: Solomon S, Qin D, Manning M, et al. Climate change 2007: the physical science basis, contribution of working group I to the fourth assessment report of the intergovernmental panel on climate change. Cambridge, UK: Cambridge University Press; 2007. pp. 499-587.

2. Wuebbles DJ, Hayhoe K. Atmospheric methane and global change. Earth Sci Rev 2002;57:177-210.

3. Patra AK. Enteric methane mitigation technologies for ruminant livestock: a synthesis of current research and future directions. Environ Monit Assess 2012;184:1929-52.

4. Grainger C, Beauchemin KA. Can enteric methane emissions from ruminants be lowered without lowering their production. Anim Feed Sci Technol 2011;166-67:308-20.

5. Kamra DN, Agarwal N, Chaudhary LC. Inhibition of ruminal methanogenesis by tropical plants con- taining secondary compounds. Int Congr Ser 2006;1293:156-63.

6. MacArtain P, Gill CIR, Brooks M, et al. Nutritional value of edible seaweeds. Nutr Rev 2007;65:535-43.

7. Chopin T, Sawhney M. Seaweeds and their mariculture. In: Steele JH, Thorpe SA, Turekian KK, editors. The encyclopedia of ocean sciences. Oxford, UK: Elsevier; 2009. pp. 4477-87.
8. Paul N, Tseng CK. Seaweed. In: Lucas JS, Southgate PC, editors. Aquaculture: farming aquatic animals and plants, 2nd edition. Oxford, UK: Blackwell publishing Ltd.; 2012. pp. 268-84.

9. Chowdhury S, Huque K, Khatun M. Algae in animal production. Agracultural Science of Biodiversity and Sustainability Workshop, Tune Landboskole, Denmark; 1995. pp. 3-7.

10. Bozic A, Anderson R, Carstens G, et al. Effects of the methane-inhibitors nitrate, nitroethane, lauric acid, Lauricidin ${ }^{\circledR}$ and the Hawaiian marine algae Chaetoceros on ruminal fermentation in vitro. Biore Technol 2009;100:4017-25.

11. Plaza M, Cifuentes A, Ibanez E. In the search of new functional food ingredients from algae. Trends Food Sci Technol 2008;19:31-9.

12. Holdt SL, Kraan S. Bioactive compounds in seaweed: functional food applications and legislation. J Appl Phycol 2011;23:543-97.

13. Denman SE, McSweeney CS. Development of a Real-Time PCR assay for monitoring anaerobic fungal and cellulolytic bacterial populations within the rumen. FEMS Microbiol Ecol 2006;58:572-82.

14. Skillman LC, Toovey AF, Williams AJ, et al. Development and validation of a real-time PCR method to quantify rumen protozoa and examination of variability between Entodinium populations in sheep offered a hay-based diet. Appl Environ Microbiol 2006;72:200-6.

15. Denman SE, Tomkins NW, McSweeney CS. Quantitation and diversity analysis of ruminal methanogenic populations in response to the antimethanogenic compound bromochloromethane. FEMS Microbiol Ecol 2007;62:313-22.

16. Koike S, Kobayashi Y. Development and use of competitive PCR assays for the rumen cellulolytic bacteria: Fibrobacter succinogenes, Ruminococcus albus and Ruminococcus flavefaciens. FEMS Microbiol Ecol 2001;204:361-6.

17. SAS Institute Inc. SAS/STAT user's guide: version 9.2 edn. Cary, NC, USA: SAS Institute Inc.; 2002.

18. Ha JK, Lee SS, Moon YS, et al. Ruminant nutrition and physiology. Seoul, Korea: Seoul National University Press; 2005.

19. Denis C, Morançais M, Li M, et al. Study of the chemical composition of edible red macroalgae Grateloupia turuturu from Brittany (France). J Food Chem 2010;119:913-7.

20. Dubois B, Tomkins NW, Kinley RD, et al. Effect of tropical algae as additives on rumen in vitro gas production and fermentation characteristics. Am J Plant Sci 2013;4:34-43.

21. Kim ET, Lee SJ, Guan LL, et al. Effects of flavonoid-rich plant extracts on in vitro ruminal methanogenesis, microbial populations and fermentation characteristics. Asian-Australas J Anim Sci 2015;28:530-7.

22. Ntaikou I, Gavala HN, Kornaros M, et al. Hydrogen production from sugars and sweet sorghum biomass using Ruminococcus albus. Int J Hydrogen Energy 2008;33:1153-63.

23. Latham MJ, Wolin MJ. Fermentation of cellulose by Ruminococcus flavefaciens in the presence and absence of Methanobacterium ruminantium. Appl Environ Microbiol 1977;34:297-301.

24. Chaucheyras-Durand F, Masséglia S, Fonty G, et al. Influence of the composition of the cellulolytic flora on the development of hydrogenotrophic microorganisms, hydrogen utilization, and methane production in the rumens of gnotobiotically reared lambs. Appl 
Environ Microbiol 2010;76:7931-7.

25. Mitsumori M, Sun W. Control of rumen microbial fermentation for mitigating methane emissions from the rumen. Asian-Australas J Anim Sci 2008;21:144-54.

26. Martin C, Morgavi DP, Doreau M. Methane mitigation in ruminants: from microbe to the farm scale. Anim 2010;4:351-65.

27. Davyt D, Fernandez R, Suescun L, et al. New sesquiterpene derivatives from the red alga Laurencia scoparia. Isolation, structure determi- nation, and anthelmintic activity. J Nat Prod 2001;64:1552-5

28. Cabrita MT, Vale C, Rauter AP. Halogenated compounds from marine algae. Mar Drugs 2010;8:2301-17.

29. Mehrez AZ, Ørskov ER, Mcdonald I. Rates of rumen fermentation in relation to ammonia concentration. Br J Nutr 1977;38:437-43.

30. Larsen M, Kristensen NB. Effect of abomasal glucose infusion on splanchnic amino acid metabolism in periparturient dairy cows. J Dairy Sci 2009;92:3306-18. 